\title{
Analysis and Optimization of Tendinous Actuation for Biomorphically Designed Robotic Systems.
}

\author{
Antonio Bicchi, Domenico Prattichizzo
}

\begin{abstract}
We present a general framework for modeling a class of mechanical systems for robotic manipulation, consisting of articulated limbs with redundant tendinous actuation and unilateral constraints. Such systems, that include biomorphically designed devices, are regarded as a collection of rigid bodies, interacting through connections that model both joints and contacts with virtual springs. Methods previously developed for the analysis of force distribution in multiple whole-limb manipulation are generalized to this broader class of mechanisms, and are shown to provide a basis for the control of co-contraction and internal forces that guarantee proper operation of the system. In particular, in the presence of constraints such as those due to limited friction between surfaces or object fragility, the choice of tendon tensions is crucial to the success of manipulation. An algorithm is described that allows to evaluate efficiently set-points for the control of tendon actuators that "optimally" (in a sense to be described) comply with the given constraints.
\end{abstract}

\section{INTRODUCTION}

The articular and tendinous structure of animal limbs provide an outstanding example of mechanical systems with extremely high performance, that attracts the interest of researchers in physiology, biomaterials, and robotics. The extremely low friction in articular joints and the remotization of actuators made possible by tendon structures are two of the prominent advantages of biomorphic structures over conventional mechanical design. Besides the exceptional lubrication properties of synovial fluid, low friction in articular joints is achieved through the use of rolling pairs between bone processes. The large redundancy of the tendinous system allows actuators (muscles) to be located far away from articular joints, and offers the possibility of "co-contracting" the limbs so as to optimally tune their stiffness and configure the limbs for different tasks (precision movements, force exertion, etc.)

The analysis of both the kinematics of articular joints and the redundancy in tendinous actuation offer non-trivial difficulties. Among these are the non-holonomy of rolling pairs, and the unilateral action of tendons (no "pushing" is allowed). The literature on tendon-actuated mechanisms is relatively rich in robotics, where they have been used especially in the design of dextrous robotic hands. The necessity to avoid tendon cables to go slack has been often solved most simply by using two tendons per joint in pushpull (or agonistic-antagonistic) configuration [1]. The use of $2 n$ cables and actuators for $n$ joints however hampers

A. Bicchi is with the Interdept. Research Center "E. Piaggio", University of Pisa, Italy and the Dept. of Electrical Systems and Automation, University of Pisa, Italy. E-mail: bicchi@ing.unipi.it

Domenico Prattichizzo is with Dipartimento di Ingegneria dell'Informazione, Università di Siena, Italy. E-mail: domenico@ing.unisi.it the practicality of the design and affects its cost. In [2] and [3], authors described the use of co-contraction in mechanisms using $n+1$ tendons. Among the novel applications of tendon actuation systems, are those to minimally-invasive surgery [4], and to haptic displays [5].

A general analysis of tendon driven mechanisms has been attempted in most cases for systems where tendons are routed through joints by means of pulleys (see e.g. [6]). A qualitative description of more general systems composed of nets of tendons and actuators has been presented by Barbieri and Bergamasco [7]. In [8] authors modeled a tendon as a transmission line. Although the tendon model is realistic, its integration into the complete dynamics of the manipulator results to be complex. Shen et al. [9] studied manipulation forces in tendon-driven manipulation systems, while Kurtz and Hayward [10] studied dexterity measures under the unilateral constraint implied by tendons. In [11], authors discussed dynamics of a 1 DOF tendon-driven manipulator and provided a parameter sensitivity analysis. Kobayashi et al. [12] developed a redundant 2-DOF manipulator with 6 tendons and showed that the mechanism safely works even if 3 tendons are broken. Kobayashi et al. [13] analyzed structural properties of mechanisms with a kinematically redundant set of tendons, and showed that a redundant number of tendons allows to adjust the manipulator stiffness and increase the mechanims fault tolerance. Prisco and Bergamasco [14] investigated the dynamics of a multi DOF tendon-driven manipulator taking into account viscoelasticity of tendons, inertia and transmission kinematics.

More complex models of tendon-actuated systems have been considered in the biomechanical literature [15], [16], [17], [18], [19]. Some authors used anthropomorphic models to attack the design of robot hands [20], [21], [22], [23].

The approach proposed in this paper is meant to encompass a wide variety of configurations that can be encountered in biological systems or conceived for artificial devices. For the sake of the widest generality, we model articulated limbs with tendinous actuation and manipulated objects as a collection of rigid bodies, interacting through contacts with characteristic kinematic and viscoelastic properties [24]. Distinction between manipulator "links" and "objects" to be manipulated is not intrinsic to the model, but can be recovered in the final stage of analysis. Contacts of any of the bodies with others are allowed, so that whole-limb manipulation is naturally investigated in this framework. Also, kinematic rolling pairs are allowed not only between a link and the manipulated object, but also between links in the same limb chain, so as to permit the above mentioned, high-efficiency biomorphic joints to 


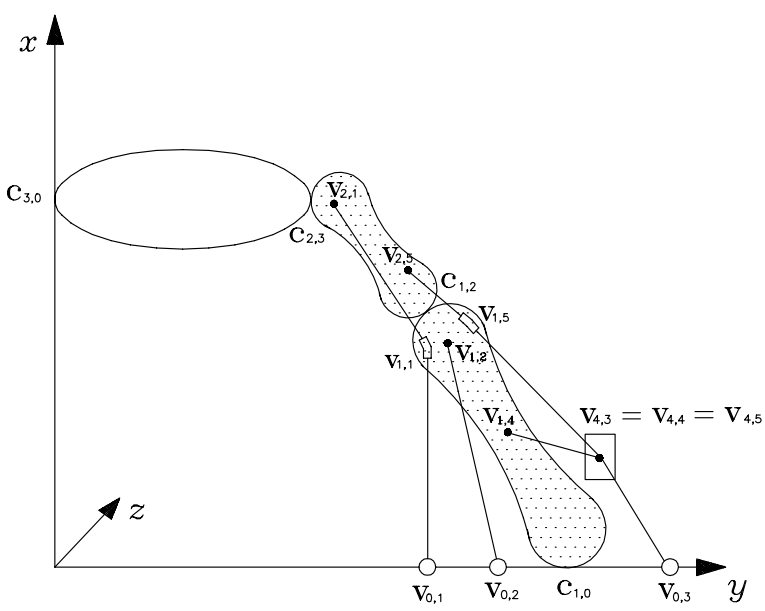

Fig. 1. A finger with two phalanges and three tendons manipulating an object against a wall.

be included in the analysis.

A quasi-static, small-displacement analysis of force distribution between contacts and through tendons for these systems is proposed that follows the lines of [25]. As a result of the proposed analysis, one is able to describe how external and internal forces are distributed in the system in a given configuration. Internal forces are defined (as usual in grasp literature) as the set of tendon tensions and contact forces that are self-balanced, i.e., do not affect motions of any part of the system. Among internal forces, "cocontraction" forces are further distinguished as those combinations of tendon tensions that influence contact forces between finger links, but do not act upon manipulated objects. The actual capability of the system to actively control such forces is investigated. By these means, a basis for the choice of internal forces that allow to avoid loosing the grip on manipulated objects, as well as a basis for co-contraction forces to avoid tendon backlash and joint disruption, is provided. The actual choice of actuating actions on the system can be eventually made based on an optimality criterion that embodies some measure of the distance between the present configuration of forces and tensions and the constraint boundaries. An implementation of such an approach is described in this paper, along with an algorithm that allows to efficiently implement the optimal choice. A proof of the global asymptotic convergence of the algorithm to the desired optimum value is given. Finally, the proposed analysis method and optimization algorithm are illustrated in an example of a finger-like manipulating system.

\section{System Description}

We consider tendon-actuated robotic structures as systems comprised of an arbitrary number $n$ of rigid objects that may be connected one with any of the others, and/or with the environment, through contacts or rotoidal or prismatic joints. An example of a tendon-driven robotic manipulator is reported in fig.1. Bodies are numbered from 1 to $n$, while the environment is assigned the index 0 . Let

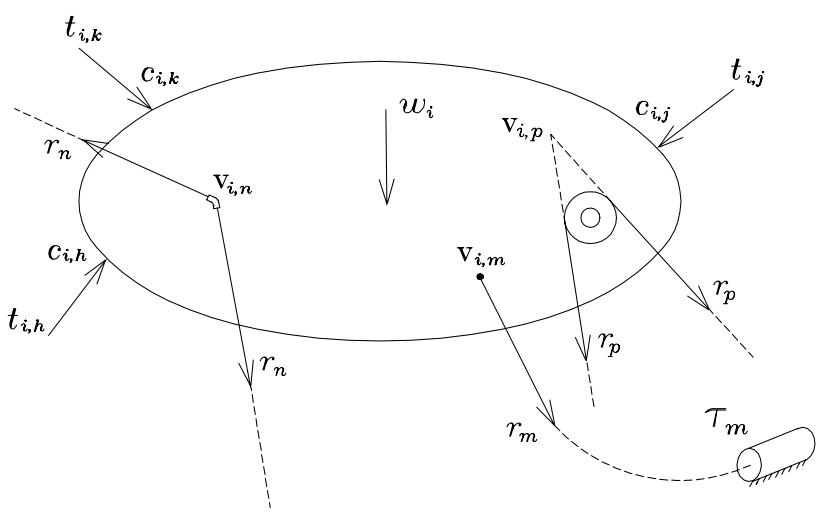

Fig. 2. Characteristic connection points, tendon conduits, and forces applied on the $i$-th body.

the location in base frame of the characteristic point of the connection between the $i$-th and $j$-th objects be $\mathbf{c}_{i, j} \in \mathbb{R}^{3}$. For prismatic or rotoidal joints, the characteristic point can be chosen as the joint origin in usual Denavit-Hartenberg conventions, while the contact centroid definition of [26] is used for contacts.

The actuation system is comprised of $q$ motors and $r$ tendons. Tendons always have one end fixed to one of the objects, while the other end may be connected to a motor or to another object $(r \geq q)$. Tendons may be routed through idle pulleys or sheaths, possibly fixed to some of the bodies, cf. fig.II. The point on body $i$ where tendon $j$ is fixed or is passed through is $\mathbf{v}_{i, j}$. According to the indexing of bodies, the position of the $j$-th tendon actuator is denoted by $\mathbf{v}_{0, j}$. Tendons are supposed uniformly stressed (frictionless transmission), and tensions applied to each tendon are collected in a vector $\mathbf{r}=\left(r_{1}, r_{2}, \ldots, r_{r}\right)^{T} \in \mathbb{R}^{r}$. We introduce the shorthand notation $\mathbf{r}=\left[r_{i}\right]_{1}^{r}$ for similar juxtaposed vectors and matrices to be encountered.

Denote with $\tilde{\mathbf{t}}_{i, j} \in \mathbb{R}^{6}$ the force/torque (wrench) exerted on the $i$-th object by the $j$-th object. Further, let $\tilde{\mathbf{t}}_{i} \in \mathbb{R}^{6 n}$ contains all vectors $\tilde{\mathbf{t}}_{i, j}$ with $j \neq i, \tilde{\mathbf{t}}_{i}=\left[\tilde{\mathbf{t}}_{i, j}\right]_{1, j \neq i}^{n}$, and let the external wrench applied on the $i$-th object be $\mathbf{w}_{i} \in \mathbb{R}^{6}$ (see fig.II). Balance equation for the $i$-th body can be written in matrix form as

$$
\mathbf{w}_{i}=-\tilde{\mathbf{G}}_{i} \tilde{\mathbf{t}}_{i}-\mathbf{T}_{i} \mathbf{r}
$$

where

$$
\begin{aligned}
\tilde{\mathbf{G}}_{i} & =\left[\tilde{\mathbf{G}}_{i, j}^{T}\right]_{1, j \neq i}^{T n} \in \mathbb{R}^{6 \times 6 n} \\
\mathbf{T}_{i} & =\left[\mathbf{T}_{i, j}^{T}\right]^{T}{ }_{1}^{r} \in \mathbb{R}^{6 \times r}
\end{aligned}
$$

being $\mathbf{T}_{i, j}$ described in the appendix and $\tilde{\mathbf{G}}_{i, j}=$ $\left(\begin{array}{cc}\mathbf{I}_{3} & \mathbf{0} \\ \mathbf{c}_{i, j} \times & \mathbf{I}_{3}\end{array}\right) \in \mathbb{R}^{6 \times 6}$ if the $i$-th object is in contact with the $j$-th object, or $\tilde{\mathbf{G}}_{i, j}=\mathbf{0}_{6 \times 6}$ otherwise.

The overall system balance equation can be written as

$$
\mathbf{w}=-\tilde{\mathbf{G}} \tilde{\mathbf{t}}-\operatorname{Tr}
$$

where $\mathbf{w}=\left[\mathbf{w}_{i}\right]_{1}^{n} \in \mathbb{R}^{6 n}, \tilde{\mathbf{t}}=\left[\tilde{\mathbf{t}}_{i}\right]_{1}^{n} \in \mathbb{R}^{6 n^{2}}$, and

$$
\begin{aligned}
& \tilde{\mathbf{G}}=\operatorname{diag}\left[\tilde{\mathbf{G}}_{i}\right]_{1}^{n} \in \mathbb{R}^{6 n \times 6 n^{2}} \\
& \mathbf{T}=\left[\mathbf{T}_{i}\right]_{1}^{n} \in \mathbb{R}^{6 n \times r} .
\end{aligned}
$$


To take into account that not all tendons are directly actuated, a suitable selection matrix $\Gamma \in \mathbb{R}^{q \times r}$ which maps tendon tensions $\mathbf{r}$ to vector of forces $\tau$ applied on tendons by the $q$ motors is introduced as

$$
\tau=\Gamma \mathbf{r}
$$

where $\Gamma_{i, j}=1$ if the $j$-th tendon is directly connected to the $i$-th motor, and $\Gamma_{i, j}=0$ otherwise.

To model constraints due to joints, and to contacts between bodies, consider two reference frames $C_{i, j}$ and $C_{j, i}$ fixed with the object $i$ and the object $j$, respectively, and centered in $\mathbf{c}_{i, j}$. Corresponding to a small displacement $\Delta \mathbf{l}_{i}$ and rotation $\Delta \Phi_{i}$ of the $i$-th object (summarized in the twist vector $\left.\Delta \mathbf{u}_{i}=\left(\Delta \mathbf{l}_{i}^{T}, \Delta \Phi_{i}^{T}\right)^{T} \in \mathbb{R}^{6}\right)$, frame $C_{i, j}$ is displaced by $\Delta \mathbf{v}_{i, j}$ and rotated by $\Delta \phi_{i, j}$

$$
\Delta \mathbf{x}_{i, j}=\left(\begin{array}{c}
\Delta \mathbf{v}_{i, j} \\
\Delta \phi_{i, j}
\end{array}\right)=\tilde{\mathbf{G}}_{i, j}^{T} \Delta \mathbf{u}_{i}
$$

and analogously for $\Delta \mathbf{x}_{j, i}$. Connection (joint or contact) constraints impose that some components of the relative displacement $\Delta \mathbf{x}_{i, j}-\Delta \mathbf{x}_{j, i}$ are opposed by reaction forces while others are left free, depending upon the type of connection. The structure of the connection between the $i-$ th and $j$-th bodies is summarized by a selection matrix, $\mathbf{H}_{i, j} \in \mathbb{R}^{t_{i j} \times 6}$ such that forces and torques mutually exerted at the connection are written as

$$
\mathbf{t}_{i, j}=\mathbf{K}_{i, j} \mathbf{H}_{i, j}\left(\Delta \mathbf{x}_{i, j}-\Delta \mathbf{x}_{j, i}\right)+\hat{\mathbf{t}}_{i, j}
$$

where the stiffness matrix $\mathbf{K}_{i, j} \in \mathbb{R}^{t_{i, j} \times t_{i, j}}$ incorporates the structural elasticity of the connection elements ("virtual springs"), and $\hat{\mathbf{t}}_{i, j}$ is the contact force when the relative displacement is zero. Note that in eq. (2), $\mathbf{t}_{i, j} \in \mathbb{R}^{t_{i j}}$ differs from $\tilde{\mathbf{t}}_{i, j}=\mathbf{H}_{i, j}^{T} \mathbf{t}_{i, j} \in \mathbb{R}^{6}$ in that only the components of the connection wrench that are relevant to the interaction are present. The $n^{2}$ equation (2) can be summarized, using eq. (1), as

$$
\mathbf{t}=\mathbf{K H X} \Delta \mathbf{x}+\hat{\mathbf{t}}=\mathbf{K H X} \tilde{\mathbf{G}}^{T} \Delta \mathbf{u}+\hat{\mathbf{t}}
$$

where $\Delta \mathbf{x}=\left[\Delta \mathbf{x}_{i}\right]_{1}^{n}$ and $\Delta \mathbf{x}_{i}=\left[\Delta \mathbf{x}_{i, j}\right]_{1, j \neq i}^{n}$. Matrix $\mathbf{X} \in \mathbb{R}^{6 n^{2} \times 6 n^{2}}$ selects appropriate combinations of displacements $\Delta \mathbf{x}_{i, j}$. The structure of $\mathbf{H} \in \mathbb{R}^{s \times 6 n^{2}}, \mathbf{K} \in$ $\mathbb{R}^{s \times s}$, and $\mathbf{X}$ is described in the appendix.

The model of tendon elasticity is obtained introducing the tendon relative displacement $\Delta \mathbf{x}_{r}$ and the displacements imposed by motors on tendon ends, $\Delta \mathbf{q}$. Accordingly, the tension of tendons $\mathbf{r}$ is

$$
\mathbf{r}=\mathbf{K}_{r} \Delta \mathbf{x}_{r}+\hat{\mathbf{r}}=\mathbf{K}_{r}\left(\mathbf{T}^{T} \Delta \mathbf{u}-\Gamma^{T} \Delta \mathbf{q}\right)+\hat{\mathbf{r}}
$$

where $\hat{\mathbf{r}}$ are the tensions in the reference configuration $\Delta \mathbf{x}_{r}=0$. The diagonal stiffness matrix $\mathbf{K}_{r} \in \mathbb{R}^{r \times r}$ depends on the elastic characteristics of tendons.

According to the above definitions and notations, the model of the system to be studied can be summarized by the following equations:

$$
\begin{aligned}
\mathbf{w} & =-\tilde{\mathbf{G}} \tilde{\mathbf{t}}-\mathbf{T r}=-\left(\tilde{\mathbf{G}} \mathbf{H}^{T} \mathbf{T}\right)\left(\begin{array}{l}
\mathbf{t} \\
\mathbf{r}
\end{array}\right) \stackrel{\text { def }}{=}-\overline{\mathbf{G}} \overline{\mathbf{t}} \\
\tau & =\Gamma \mathbf{r} \\
\mathbf{t} & =\mathbf{K} \mathbf{H X} \Delta \mathbf{x}+\hat{\mathbf{t}}=\mathbf{K} \mathbf{H X} \tilde{\mathbf{G}}^{T} \Delta \mathbf{u}+\hat{\mathbf{t}} \\
\mathbf{r} & =\mathbf{K}_{r} \Delta \mathbf{x}_{r}+\hat{\mathbf{r}}=\mathbf{K}_{r}\left(\mathbf{T}^{T} \Delta \mathbf{u}-\Gamma^{T} \Delta \mathbf{q}\right)+\hat{\mathbf{r}}
\end{aligned}
$$

\section{Force Distribution}

The force distribution problem consists of describing the general solution to eq. (3), a linear system of $6 n$ equation in $s+r$ unknowns. Note that eq. (3) admits solution only if $\mathbf{w}$ belongs to the range space of $\overline{\mathbf{G}}$. A wrench outside such range could not be counterbalanced by any actuator action: this situation is usually avoided by design. Thus, we assume that $\mathcal{R}(\overline{\mathbf{G}})=\mathbb{R}^{6 n}$. The general solution of eq. (3) can be written as the sum of a particular solution and an homogeneous solution.

\section{A. Particular solution}

The particular solution is in general not unique, since $\overline{\mathbf{G}}$ admits infinitely many right inverses. However, we expect a unique solution to the following problem:

Assume the system is in an equilibrium configuration, under a set of external loads $\hat{\mathbf{w}}$, with contact forces $\hat{\mathbf{t}}$ and tensions of tendons $\hat{\mathbf{r}}$. Determine the contact forces and tendon tensions at the equilibrium that the system reaches when an additional load $\mathbf{w}$ is applied, while the actuator position $\mathbf{q}$ is kept constant.

Eq.(5) and (6) with $\Delta \mathbf{q}=0$, can be rewritten as follows

$$
\overline{\mathbf{t}}=\left(\begin{array}{l}
\mathbf{t} \\
\mathbf{r}
\end{array}\right)=\left(\begin{array}{cc}
\mathbf{K} & 0 \\
0 & \mathbf{K}_{r}
\end{array}\right)\left(\begin{array}{c}
\mathbf{G}_{x}^{T} \\
\mathbf{T}^{T}
\end{array}\right) \Delta \mathbf{u}+\left(\begin{array}{c}
\hat{\mathbf{t}} \\
\hat{\mathbf{r}}
\end{array}\right) \stackrel{\text { def }}{=} \overline{\mathbf{K}} \overline{\mathbf{G}}_{x}^{T} \Delta \mathbf{u}+(\hat{\overline{\mathbf{t}}})
$$

where $\mathbf{G}_{x}=\mathbf{H X} \tilde{\mathbf{G}}^{T}$. Substituting eq.(7) in eq.(3), we have

$$
\mathbf{w}+\hat{\mathbf{w}}=-\overline{\mathbf{G}} \overline{\mathbf{K}} \overline{\mathbf{G}}_{x}^{T} \Delta \mathbf{u}-\overline{\mathbf{G}} \hat{\overline{\mathbf{t}}}
$$

Hence, being $\hat{\mathbf{w}}=-\overline{\mathbf{G}} \hat{\overline{\mathbf{t}}}$ and recalling that $\overline{\mathbf{G}}$ is assumed full row rank and $\overline{\mathbf{K}}$ is invertible, one gets

$$
\overline{\mathbf{t}}=\left(\begin{array}{c}
\mathbf{t} \\
\mathbf{r}
\end{array}\right)=-\overline{\mathbf{K}} \overline{\mathbf{G}}_{x}^{T}\left(\overline{\mathbf{G}} \overline{\mathbf{K}} \overline{\mathbf{G}}_{x}^{T}\right)^{-1} \mathbf{w}+\hat{\overline{\mathbf{t}}} \stackrel{\text { def }}{=}-\overline{\mathbf{G}}_{K}^{R} \mathbf{w}+\hat{\overline{\mathbf{t}}}
$$

where $\overline{\mathbf{G}}_{K}^{R}$ is the $\overline{\mathbf{K}}$-weighted right inverse of $\overline{\mathbf{G}}$. It ensues that the particular solution for tendon tensions and contact forces between any pair of bodies caused by the external wrench w can be easily recovered from (8).

\section{B. Homogeneous solution}

Homogeneous solutions of eq. (3) correspond to tendon tensions and connection forces that counterbalance each other, thus not affecting the overall equilibrium of the system. These forces and tensions are usually referred to as internal. Internal forces are of fundamental concern in grasp planning, since disruption of rolling-pair joints or slippage 
and loss of grasp stability can often be avoided only through effective management of internal forces. In analogy with systems of whole-limb manipulators without tendons discussed in [25], in the present case we may be confronted with the impossibility of arbitrarily controlling every combination of internal forces in the nullspace of $\overline{\mathbf{G}}$. Among internal tensions-forces, $\mathrm{co}^{-}$-contraction tendon tensions can be further distinguished as those that do not affect contact forces between the links and the manipulated objects. Co-contraction tensions must be used to keep tensions positive in each tendon (to avoid them to go slack), and to keep contact between different links in the limbs (when e.g. a rolling-pair joint is used).

\section{B.1 Active internal tensions and forces}

Let us rewrite eq.(5) and (6) as $\overline{\mathbf{t}}=\left(\begin{array}{c}\mathbf{t} \\ \mathbf{r}\end{array}\right)=\left(\begin{array}{cc}\mathbf{K G}_{x}^{T} & 0 \\ \mathbf{K}_{r} \mathbf{T}^{T} & -\mathbf{K}_{r} \Gamma^{T}\end{array}\right)\left(\begin{array}{c}\Delta \mathbf{u} \\ \Delta \mathbf{q}\end{array}\right) \stackrel{\text { def }}{=} \mathbf{M}\left(\begin{array}{c}\Delta \mathbf{u} \\ \Delta \mathbf{q}\end{array}\right)$

We now prove that every active internal (contact and tendon) force can be written as the product of a basis matrix $\mathbf{E}$ times an arbitrary coefficient vector $\mathbf{y}$ of suitable dimension. In fact, consider an equilibrium configuration of the system under the wrench $\hat{\mathbf{w}}$ and let $\hat{\overline{\mathbf{t}}}, \hat{\mathbf{q}}$ be the connection/tendon forces and positions of tendon actuated ends, respectively. Let $\delta \mathbf{u}$ be a vector containing virtual displacements of the objects compatible with all connection constraints. Applying the principle of virtual work (PVW) and eq.(3), we have

$$
\hat{\mathbf{w}}^{T} \delta \mathbf{u}=\hat{\overline{\mathbf{t}}}^{T} \overline{\mathbf{G}}^{T} \delta \mathbf{u}=0, \forall \delta \mathbf{u} .
$$

Perturb the equilibrium configuration by imposing displacements of the actuated ends of tendons by $\Delta \mathbf{q}$, and let $\Delta \overline{\mathbf{t}}, \Delta \mathbf{u}$ be the change of tensions and forces and the change of position of bodies, respectively. A new equilibrium configuration, under the same set of external forces $\hat{\mathbf{w}}$, will be reached on condition that the PVW is satisfied:

$$
\hat{\mathbf{w}}^{T} \delta \mathbf{u}=\left(\hat{\overline{\mathbf{t}}}^{T}+\Delta \overline{\mathbf{t}}^{T}\right) \overline{\mathbf{G}}^{T} \delta \mathbf{u}=\Delta \overline{\mathbf{t}}^{T} \overline{\mathbf{G}}^{T} \delta \mathbf{u}=0, \quad \forall \delta \mathbf{u} .
$$

Substituting eq.(III-B.1), the PVW condition is rewritten as

$$
\overline{\mathbf{G}} \mathbf{M}\left(\begin{array}{c}
\Delta \mathbf{u} \\
\Delta \mathbf{q}
\end{array}\right)=0
$$

Let $\mathbf{B} \in \mathbb{R}^{(6 n+q) \times b}$ be a matrix whose columns span the nullspace of $\overline{\mathbf{G} M}$. According to (III-B.1), the subspace of internal forces that can be obtained at steady-state after a displacement of the tendon ends is commanded, i.e. the subspace of active internal forces $\mathcal{F}_{h a}$, is given by the range space of $\mathbf{M B}$ whose basis matrix $\mathbf{E} \in \mathbb{R}^{s \times e}$ is obtained by using only the independent columns of the product $\mathbf{M B}$. Therefore,

$$
\mathcal{F}_{h a}=\left\{\overline{\mathbf{t}}: \mathbf{E y}, \mathbf{y} \in \mathbb{R}^{e}\right\} .
$$

The basis matrix $\mathbf{E}$ can be partitioned as $\mathbf{E}=\left(\left[\mathbf{E}_{i}\right]_{1}^{n} \mathbf{E}_{r}\right)$, with $\mathbf{E}_{i}=\left[\mathbf{E}_{i, j}\right]_{1, j \neq i}^{n}$. Blocks $\mathbf{E}_{i, j} \in \mathbb{R}^{t_{i, j} \times e}$ correspond to contact forces between the $i$-th and the $j$-th body contributing to active internal forces, while block $\mathbf{E}_{r}$ contains the corresponding tensions of tendons necessary to apply the internal forces. Note that the set of the " $\mathrm{CO}^{-}$ contraction" forces $\mathcal{F}_{c c}$ is the subspace of active internal forces that do not change the forces between links and the manipulated object:

$\mathcal{F}_{c c}=\left\{\overline{\mathbf{t}}: \overline{\mathbf{t}}=\mathbf{E y}, \mathbf{y} \in \mathbb{R}^{e}, \mathbf{E}_{i, j} \mathbf{y}=0\right.$, if body $i$ or $j$ is the "object

\section{B.2 Preload internal forces}

We consider preload tensions and forces as those internal tensions and forces that can not be actively controlled by means of motor displacements. Preloading a system can avoid slippage due to a low value of non controllable normal forces. In the analysis of systems with tendinous structure and rolling pairs, preloading can be used to model the effects of articular ligaments.

Letting $\Gamma_{T}=(\mathbf{0} \Gamma) \in \mathbb{R}^{q \times(s+r)}$, eq. (4) is rewritten as $\tau=\Gamma_{T} \overline{\mathbf{t}}$ and eq. (5) and (6) can be assembled as

$\overline{\mathbf{t}}=\left(\begin{array}{c}\mathbf{t} \\ \mathbf{r}\end{array}\right)=\left(\begin{array}{cc}\mathbf{K} \mathbf{H}_{x} & \mathbf{0} \\ \mathbf{0} & \mathbf{K}_{r}\end{array}\right)\left(\begin{array}{c}\Delta \mathbf{x} \\ \Delta \mathbf{x}_{r}\end{array}\right) \stackrel{\text { def }}{=} \mathbf{K}^{\prime}\left(\begin{array}{c}\Delta \mathbf{x} \\ \Delta \mathbf{x}_{r}\end{array}\right)$.

Because every preload force is internal and not controllable by motors, the following relations must be verified

$$
\begin{gathered}
\overline{\mathbf{G}} \mathbf{K}^{\prime}\left(\begin{array}{c}
\Delta \mathbf{x} \\
\Delta \mathbf{x}_{r}
\end{array}\right)=\mathbf{0} \\
\Gamma_{T} \mathbf{K}^{\prime}\left(\begin{array}{c}
\Delta \mathbf{x} \\
\Delta \mathbf{x}_{r}
\end{array}\right)=\mathbf{0}
\end{gathered}
$$

The subspace of preload tensions and forces $\mathcal{F}_{h p}$ is therefore given by

$$
\mathcal{F}_{h p}=\left\{\overline{\mathbf{t}}: \overline{\mathbf{t}}=\mathbf{K}^{\prime} \mathbf{y}, \mathbf{y} \in \mathcal{N}\left(\overline{\mathbf{G}} \mathbf{K}^{\prime}\right) \cap \mathcal{N}\left(\Gamma_{T} \mathbf{K}^{\prime}\right)\right\}
$$

where $\mathcal{N}(\cdot)$ indicates the nullspace of the argument.

\section{Optimization OF TENDON TENSIONS}

In this section, we assume for simplicity that the system is not preloaded (this assumption implies no loss of generality, as preload effects could be easily taken into account by superimposing them to the effects of external wrenches). The general solution of eq.(3) can be written as

$$
\overline{\mathbf{t}}=\overline{\mathbf{G}}_{K}^{R} \mathbf{w}+\mathbf{E y} .
$$

where $\mathbf{y}$ is a free vector parameterizing the internal tensions/forces (including tendinous tensions used in cocontraction), which may be chosen according to the system constraints, among which the most important are typically those listed in the following.

Maximum contact forces A delicate object could be damaged by too large forces; in some cases, it is some parts of the robot system (e.g. the force sensors) that might be hurt. A safety threshold, depending on the objects involved in the contact interaction, should be chosen to limit the intensity of contact forces. These bounds can be summarized as

$\left\|\mathbf{p}_{i, j}\right\| \leq f_{i, j, \max }>0, i=1,2, \ldots, n, j=0,2, \ldots, n, i \neq j$. 
where $\mathbf{p}_{i j}$ is the contact force exerted on the $i$-th object by the $j$-th object and $\|\cdot\|$ indicates the euclidean norm of the argument.

Minimum contact forces There are also reasons to keep contact forces above a minimum value. One is of practical nature: contact sensors work better in a certain range of forces, and cannot distinguish too small forces from noise. Another, perhaps deeper, reason is that one would like to avoid the temporal discontinuity of contacts. Klein and Kittivatcharapong [1990] designed with this term a phenomenon, consisting in a low-frequency sequence of impacts between some links of the manipulators and the object. This highly undesirable "chattering" of the contact forces has been encountered e.g. by Cheng and Orin [1990], who explained it as due to a substantial freedom in the solution of (3) while yet meeting some underconstraining optimality criterion.

A lower bound on the normal component of contact forces can be imposed as

$\mathbf{p}_{i j}^{T} \mathbf{n}_{i, j} \geq f_{i, j, \min }>0, \quad i=1,2, \ldots, n, j=0,1, \ldots, n, i \neq j$.

where $\mathbf{n}_{i, j}$ is the unit normal vector pointing toward the surface of object $i$ at the contact point between objects $i$ and $j$.

Friction limits. In the absence of local contact torques, the normal and tangential components of each contact force $\mathbf{p}_{i, j}$ must comply with Coulomb's law of friction

$$
\mathbf{p}_{i, j}^{T} \mathbf{n}_{i, j} \geq \frac{1}{\mu_{i, j}}\left\|\left(\mathbf{I}-\mathbf{n}_{i j} \mathbf{n}_{i, j}^{T}\right) \mathbf{p}_{i, j}\right\|=\alpha_{i, j}\left\|\mathbf{p}_{i, j}\right\|,
$$

where $\mu_{i, j}$ is the static friction coefficient in the current contact conditions, and $\alpha_{i, j}=\left(1+\mu_{i, j}^{2}\right)^{-1 / 2}$.

When more realistic models of contact are assumed, friction limits involve much more complex relationships. For the case of "soft-finger" contacts [3], a simplified linear relationship can be assumed as

$\mathbf{p}_{i, j}^{T} \mathbf{n}_{i, j} \geq \alpha_{i, j}\left\|\mathbf{p}_{i, j}\right\|+\beta_{i, j}\left\|\mathbf{q}_{i, j}\right\|$

$i=1,2, \ldots, n, j$

where $\alpha_{i, j}=\left(1+\mu_{i, j}^{2}\right)^{-1 / 2}$ and $\beta_{i, j}=1 / \mu_{i, j}$, and $\mu_{i, j}$ is the rotational friction coefficient. For a more accurate discussion of related questions, see e.g. [Goyal, 1989].

Maximum tendon tension. The tendon stress amplitude must be less then a maximum fixed value. This value depends of the made of tendons. We have:

$$
r_{i} \leq r_{i, \max } \quad i=1,2, \ldots, r .
$$

Minimum tendon tension. The tendon stress amplitude must be grater then a minimum fixed value. We have:

$$
r_{i} \geq r_{i, \min } \quad i=1,2, \ldots, r .
$$

\section{A. Cost function}

In this subsection, we proceed to construct a cost function whose minimzation can guarantee fulfilling all constraints introduced above. Following the approach of [27], the constraints eq. (9), eq. (10), and eq. (11) relative to the contact between object $i$ and $j$, can all be written in the same form as

$\sigma_{i, j, k}(\mathbf{y}, \mathbf{w})=\alpha_{i, j, k}\left\|\mathbf{p}_{i, j}\right\|+\beta_{i, j, k}\left\|\mathbf{q}_{i, j}\right\|+\gamma_{i, j, k} \mathbf{p}_{i, j}^{T} \mathbf{n}_{i, j}+\delta_{i, j, k} \leq 0$

where $\alpha_{i, j, 1}=1, \beta_{i, j, 1}=0, \gamma_{i, j, 1}=0$, and $\delta_{i, j, 1}=-f_{i, j, \max }$ for maximum force constraints; $\alpha_{i, j, 2}=0, \beta_{i, j, 2}=0$, $\gamma_{i, j, 2}=-1$, and $\delta_{i, j, 2}=f_{i, j, m i n}$ for minimum force constraints; and $\alpha_{i, j, 3}=\alpha_{i, j}, \beta_{i, j, 3}=\beta_{i, j}, \gamma_{i, 3}=-1$, and $\delta_{i, 3}=0$ for friction constraints. Also tendon constrains can be summarized in a single expression as

$$
\nu_{i, j}(\mathbf{y}, \mathbf{w})=\rho_{i, j} r_{i}+\eta_{i, j}
$$

where $\rho_{i, 1}=-1$ and $\eta_{i, 1}=f_{i, \min }$ for minimum stress constraints; $\rho_{i, 1}=1$ and $\eta_{i, 1}=-f_{i, \max }$ for maximum stress constraints.

Let $\Omega_{i, j, k}^{\kappa} \subset \Re^{e}$ (resp., $\Gamma_{i, j}^{\kappa} \subset \Re^{e}$ ) indicate the sets of free parameters $\mathbf{y}$ that, in the presence of a given load $\mathbf{w}$, satisfy constraints in eq. (12) (resp., eq. (13)) of corresponding indices with a (small, positive) margin $\kappa$. In other words, let $\Omega_{i, j, k}^{\kappa}:=\left\{\mathbf{y} \mid \sigma_{i, j, k}(\mathbf{y})<-\kappa\right\}$ and $\Gamma_{i, j}^{\kappa}:=\left\{\mathbf{y} \mid \nu_{i, j}(\mathbf{y})<-\kappa\right\}$. Notice explicitely that the region where all constraints are satisfied, $\Omega^{0} \cap \Gamma^{0}$, with

$$
\Omega^{0} \stackrel{\text { def }}{=} \bigcap_{i, j, k} \Omega_{i, j, k}^{0}, \quad \Gamma^{0} \stackrel{\text { def }}{=} \bigcap_{i, j} \Gamma_{i, j}^{0}
$$

contains $\Omega^{\kappa} \cap \Gamma^{\kappa}$, where

$$
\Omega^{\kappa}=\bigcap_{i, j, k} \Omega_{i, j, k}^{\kappa}, \quad \Gamma^{\kappa}=\bigcap_{i, j} \Gamma_{i, j}^{\kappa}
$$

i.e., the set of parameters which fulfill constraints with a guaranteed margin $k$.

For the contact between object $i$ and $j$ and for the $k$-th constraint, consider the cost function

$$
V_{c_{i, j, k}}^{\kappa}(\mathbf{y}, \mathbf{w})=\left\{\begin{array}{cccc}
\left(2 \sigma_{i, j, k}^{2}\right)^{-1} & \mathbf{y} & \in & \Omega_{i, j, k}^{\kappa} \\
a \sigma_{i, j, k}^{2}+b \sigma_{i, j, k}+c & \mathbf{y} & \notin & \Omega_{i, j, k}^{\kappa}
\end{array},\right.
$$

For the $i$-th,$i \neq j$ tendon and $j$-th constraint consider

$$
V_{t_{i, j}}^{\kappa}(\mathbf{y}, \mathbf{w})=\left\{\begin{array}{cccc}
\left(2 \nu_{i, j}^{2}\right)^{-1} & \mathbf{y} & \in & \Gamma_{i, j}^{\kappa} \\
a \nu_{i, j}^{2}+b \nu_{i, j}+c & \mathbf{y} & \notin & \Gamma_{i, j}^{\kappa}
\end{array} .\right.
$$

An overall cost function is defined as the weighted sum

$$
V^{\kappa}(\mathbf{y}, \mathbf{w})=\sum_{i=1}^{n} \sum_{\substack{j=0 \\ j \neq i}}^{n} \sum_{k=1}^{3} w_{c_{i, j, k}} V_{c_{i, j, k}}^{\kappa}(\mathbf{y})+\sum_{i=1}^{r} \sum_{j=1}^{2} w_{t_{i, j}} V_{t_{i, j}}^{\kappa}(\mathbf{y})
$$

where $w_{c_{i, j, k}}>0$ and $w_{c_{i, j}}>0$ are positive weights. Notice that, for $k=0$, each addend $V_{c_{i, j, k}}^{\kappa}$ turns into

$$
V_{c_{i, j, k}}(\mathbf{y}, \mathbf{w})=\left\{\begin{array}{ccccc}
\left(2 \sigma_{i, j}^{2}\right)^{-1} & \mathbf{y} & \in & \Omega_{i, j, k}^{0} ; \\
\infty & \mathbf{y} & \notin & \Omega_{i, j, k}^{0} .
\end{array},\right.
$$

and similarly for $V_{t_{i, j}}^{\kappa}$. The following proposition can be shown to hold (arguments are similar to those used in [27], and are omitted here for brevity). 
Proposition 1: For $\kappa=0$, the cost function defined in eq. (16), restricted to $\Omega^{0} \cap \Gamma^{0}$, is strictly convex with respect to $\mathbf{y} \in \Re^{h}$. For $\kappa \neq 0$, with the choice $a=\frac{3}{2 \kappa^{4}}, b=\frac{4}{\kappa^{3}}$, and $c=\frac{3}{\kappa^{2}}$, the cost is twice continuously differentiable and globally strictly convex with respect to $\mathbf{y} \in \Re^{h}$.

Given the convexity of the cost functions with $\kappa \neq 0$ over the whole space of free parameters, standard techniques can be employed to search the unique minimizer

$$
\widehat{\mathbf{y}}^{\kappa}=\arg \min _{\mathbf{y}} V^{\kappa}(\mathbf{y}, \widetilde{\mathbf{w}}) .
$$

For instance, the following update law:

$$
\dot{\mathbf{y}}(t)=-\zeta \frac{\partial^{2} V^{\kappa}}{\partial y^{2}} \quad \frac{\partial V^{\kappa}}{\partial y}
$$

where

$$
\zeta=\lambda+\frac{\frac{\partial V^{\kappa} T}{\partial w} \dot{\mathbf{w}}(t)}{\frac{\partial V^{\kappa}}{\partial y}{\frac{\partial^{2} V^{\kappa}}{\partial y^{2}}}^{-1} \frac{\partial V^{\kappa}}{\partial y}}, \lambda>0 .
$$

provides a globally asymptotically convergent algorithm, in the presence of time-varying external wrenches w. A proof of convergence is straightforwardly obtained by considering the time derivative of the positive-definite Lyapunov candidate function $V^{\kappa}$

$$
\begin{aligned}
\dot{V}^{\kappa} & =\frac{\partial V^{\kappa T}}{\partial y} \dot{\mathbf{y}}+\frac{\partial V^{\kappa T}}{\partial w} \dot{\mathbf{w}} \\
& =-\zeta \frac{\partial V^{\kappa T}}{\partial y} \frac{\partial^{2} V^{\kappa}-1}{\partial y^{2}} \frac{\partial V^{\kappa}}{\partial y}+\frac{\partial V^{\kappa T}}{\partial w} \dot{\mathbf{w}} \\
& =-\lambda \frac{\partial V^{\kappa}}{\partial y} \frac{\partial^{2} V^{\kappa}}{\partial y^{2}} \frac{\partial V^{\kappa}}{\partial y} \leq 0 .
\end{aligned}
$$

Recalling that $\frac{\partial^{2} V^{\kappa}}{\partial \mathbf{y}^{2}}$ is positive definite for any $\mathbf{y} \in \Re^{h}$, the only possible equilibrium point is for $\frac{\partial V^{\kappa}}{\partial y}=0$, i.e., at the optimum.

The optimization algorithm can be efficiently implemented exploiting the simple structure of $V$ : explicit expressions for $\frac{\partial V^{\kappa}}{\partial \mathbf{y}}$ and $\frac{\partial^{2} V^{\kappa}}{\partial \mathbf{y}^{2}}$ are in fact easily obtained (compare e.g. with those reported in [27]. If an initial feasible solution is available (i.e., a parameter $\mathbf{y}(0) \in \Omega^{0} \cap \Gamma^{0}$ ), then the algorithm can be used directly with $\kappa=0$. If otherwise no such guess is available, to guarantee global asymptotic convergence of the algorithm is necessary to choose $\kappa>0$. If a minimizer is found to this problem such that $\widehat{\mathbf{y}}^{\kappa} \in \Omega_{k}$, then it follows from the discussion above that $\widehat{\mathbf{y}}^{\kappa}=\widehat{\mathbf{y}}^{0}$. If such condition is not verified, it is necessary to proceed to a new optimization with smaller $\kappa$.

Although the algorithm has been discussed in the continuous time domain, it is straightforward to derive its discrete time analog. In this case, however, the global asymptotic convergence of the algorithm can be proven only for values of $\lambda$ smaller than a limit value, whose evaluation in realtime is possible, but not easy. Such limitations on $\lambda$ will only allow the convergence to a finite neighborhood of the optimal grasp. Suitable policies for varying the step length $\lambda$ to maximize convergence rate can be realized, albeit convergence proofs may be involved in this case.

\section{EXAMPLE}

The tendon-driven manipulator of fig. 1 consists of a finger with two phalanges, pushing an object against the floor. The actuation system is comprised of three tendons, with an end fixed to a phalanx and the other connected to a motor. Two tendons go through sheaths fixed to the phalanges.

Assume the contact centroids coordinates to be (the measure unit is $\mathrm{cm}$ )

$$
\begin{gathered}
\mathbf{c}_{1,0}=\left(\begin{array}{lll}
0 & 20 & 0
\end{array}\right)^{T} ; \mathbf{c}_{1,2}=\mathbf{c}_{2,1}=\left(\begin{array}{lll}
10.4 & 14 & 0
\end{array}\right)^{T} \\
\mathbf{c}_{2,3}=\mathbf{c}_{3,2}=\left(\begin{array}{llll}
14.6 & 9.76 & 0
\end{array}\right)^{T} ; \mathbf{c}_{3,0}=\left(\begin{array}{lll}
14.6 & 0 & 0
\end{array}\right)^{T}
\end{gathered}
$$

Rolling pairs (hinge-type) contacts $\mathbf{c}_{1,0}$ and $\mathbf{c}_{1,2}$ allow the rotation only around $z$ axis, hence

$$
\mathbf{n}_{1,0}=\mathbf{n}_{1,2}=\left(\begin{array}{lll}
0 & 0 & 1
\end{array}\right)^{T} .
$$

Contacts $\mathbf{c}_{2,3}$ and $\mathbf{c}_{3,0}$ are "soft finger" type, cf. [3] with normal direction

$$
\mathbf{n}_{2,3}=\left(\begin{array}{lll}
0 & -1 & 0
\end{array}\right)^{T} ; \mathbf{n}_{3,0}=\left(\begin{array}{lll}
0 & 1 & 0
\end{array}\right)^{T},
$$

respectively. The tendon arrangment is described as follows - Tendon 1 is connected to finger 2 at $\mathbf{v}_{2,1}=(14.59 .90)^{T}$, goes through a conduit fixed to finger 1 at $\mathbf{v}_{1,1}=$ $\left(\begin{array}{ll}9.4 & 12.30\end{array}\right)^{T}$, and is connected to motor 1 at $\mathbf{v}_{0,1}=$ $\left(\begin{array}{lll}0 & 12.3 & 0\end{array}\right)^{T}$;

- Tendon 2 is connected to finger 1 and to motor 2 at $\mathbf{v}_{1,2}=\left(\begin{array}{lll}10.3 & 14.1 & 0\end{array}\right)^{T}$ and $\mathbf{v}_{0,2}=\left(\begin{array}{llll}0 & 17 & 0\end{array}\right)^{T}$;

- Tendon 3 is connected to object 4 (fictitious) at $\mathbf{v}_{4,3}=$ $(3.220 .50)^{T}$ and to motor 3 at $\mathbf{v}_{0,3}=\left(\begin{array}{l}0 \\ 0\end{array} 23.70\right)^{T}$;

- Tendon 4 is connected to object 4 (fictitious) at $\mathbf{v}_{4,4}=$ $(3.220 .50)^{T}$ and to finger 1 at $\mathbf{v}_{0,3}=(5.2170)^{T}$;

- Tendon 5 is connected to finger 2 at $\mathbf{v}_{2,5}=(10.513 .90)^{T}$, goes through a sheath fixed to finger 1 at $\mathbf{v}_{1,5}=$ $(11.415 .70)^{T}$, and is connected to object 4 (fictitious) at $\mathbf{v}_{4,5}=(3.2120 .50)^{T}$.

Dimensions of vectors and matrices are as follows: $\mathbf{t}=$ $[\mathbf{t}]_{1}^{4} \in \mathbb{R}^{37} ; \mathbf{G} \in \mathbb{R}^{24 \times 37} ; \mathbf{H} \in \mathbb{R}^{37 \times 96} ; \mathbf{K} \in \mathbb{R}^{37 \times 37} ; \mathbf{X} \in$ $\mathbb{R}^{96 \times 96} ; \mathbf{T} \in \mathbb{R}^{24 \times 5} ; \Gamma \in \mathbb{R}^{3 \times 5}$. Note that all the vectors $\mathbf{t}_{i 4}$ and $\mathbf{t}_{4 i}$ are zero because the (fictitious) link 4 is not in contact with the other links and/or the environment.

We assume a value of $100 \mathrm{~N} / \mathrm{cm}$ for stiffness of linear springs, $20 \mathrm{~N} / \mathrm{cm}$ for the stiffness of rotoidal springs, and $10 \mathrm{~N} / \mathrm{cm}$ for stiffness of tendons. Applying the algorithm shown in Section III-B.1, the basis of active internal forces is found as:

$$
\begin{gathered}
\mathbf{E}_{1,0}=\left(\begin{array}{cc}
1.9 & 0.76 \\
-1.2 & -0.45 \\
0 & 0 \\
0 & 0 \\
0 & 0
\end{array}\right) \\
\mathbf{E}_{1,2}=-\mathbf{E}_{2,1}=\left(\begin{array}{cc}
-0.29 & 0.05 \\
0.82 & 0.13 \\
0 & 0 \\
0 & 0 \\
0 & 0
\end{array}\right)
\end{gathered}
$$




$$
\begin{aligned}
& \mathbf{E}_{2,3}=-\mathbf{E}_{3,2}=\mathbf{E}_{3,0}=\left(\begin{array}{cc}
0 & 0 \\
0.23 & 0 \\
0 & 0 \\
0 & 0
\end{array}\right) \\
& \mathbf{E}_{r}=\left(\begin{array}{ll}
0.52 & 0.01 \\
0.63 & 0.63 \\
0.79 & 0.26 \\
0.40 & 0.13 \\
0.40 & 0.13
\end{array}\right) \\
& \mathbf{E}_{1,3}=\mathbf{E}_{3,1}=\mathbf{E}_{1,4}=\mathbf{E}_{4,1}=0 \\
& \mathbf{E}_{2,0}=\mathbf{E}_{2,4}=\mathbf{E}_{4,2}=\mathbf{E}_{3,4}=\mathbf{E}_{4,3}=0 \\
& \mathbf{E}_{4,0}=\mathbf{E}_{4,1}=\mathbf{E}_{4,2}=\mathbf{E}_{4,3}=0 .
\end{aligned}
$$

Note that the second column of $\mathbf{E}$ forms a basis of the active internal co-contraction forces because no forces are applied to object 3 (manipulated object). To distinguish active internal co-contraction forces from the other active internal forces a suitable algorithm can be implemented.

The preload force basis matrix $\mathbf{P}$ for this example results

$$
\begin{gathered}
\mathbf{P}_{1,0}=\mathbf{P}_{1,2}=-\mathbf{P}_{2,1}=\left(\begin{array}{ccccc}
0 & 0 & 0 & 0 & -1
\end{array}\right)^{T} \\
\mathbf{P}_{2,3}=-\mathbf{P}_{3,2}=-\mathbf{P}_{3,0}=\left(\begin{array}{llll}
0 & 0 & 0 & 1
\end{array}\right)^{T} \\
\mathbf{P}_{1,3}=\mathbf{P}_{1,4}=\mathbf{P}_{2,0}=\mathbf{P}_{2,4}=\mathbf{P}_{3,1}=\mathbf{P}_{3,4}=0 \\
\mathbf{P}_{4,0}=\mathbf{P}_{4,1}=\mathbf{P}_{4,2}=\mathbf{P}_{4,3}=0 \quad \mathbf{P}_{r}=\mathbf{0}_{5 \times 1}
\end{gathered}
$$

Allowed preload forces correspond to torsion of the object between two soft-finger contacts, and is of no concern for the tendinous actuation system.

We finally report the results of the optimization algorithm described in the text, when used to calculate the combination of internal forces to minimize the distance from violation of contact and tension constraints. An external unit force vector is applied to the centre of object 3 along the $x$-axis direction in this example. The following values of optimal contact forces and tensions have been obtained:

$$
\begin{gathered}
\mathbf{t}_{1,0}=(22.8-14.5000)^{T} ; \mathbf{t}_{1,2}=-\mathbf{t}_{2,1}=(-4.411000)^{T} \\
\mathbf{t}_{3,0}=\left(\begin{array}{ll}
-0.593 .0700 & 0
\end{array}\right)^{T} ; \mathbf{t}_{2,3}=-\mathbf{t}_{3,2}=\left(\begin{array}{lll}
0.413 .0700 & 0
\end{array}\right)^{T} \\
\mathbf{t}_{1,3}=\mathbf{t}_{3,1}=\mathbf{t}_{1,4}=\mathbf{t}_{4,1}=\mathbf{t}_{2,0}=\mathbf{t}_{2,4}=\mathbf{t}_{4,2}=0 \\
\mathbf{t}_{3,4}=\mathbf{t}_{4,3}=\mathbf{t}_{4,0}=\mathbf{t}_{4,1}=\mathbf{t}_{4,2}=\mathbf{t}_{4,3}=0 \\
r_{1}=7.8, r_{2}=4.26, r_{3}=10.02, r_{4}=5.18, r_{5}=5.18
\end{gathered}
$$

This results show that all tendons are correctly stretched, that forces at the hinges are compressive (so that a rollingpair joint can be adopted in the design), and that slippage of the object relative to the finger and to the wall is prevented.

\section{Conclusions}

We presented a method for analyzing the system of forces necessary to balance a mechanical structure, composed of rigid bodies and tendons in arbitrary combinations, and for optimizing their choice. The method is very general and allows to attack in a unified manner a very broad variety of mechanism. Although only discussed in a quasi-static setting, the method should be easily modified to model the dynamics of such systems. One of the main drawbacks of the method is the introduction of rather large matrices even for simple systems, as shown in the example above. This is a price we pay to generality, and more computationallyefficient formulations can be derived by specializing the treatment of particular cases.

\section{APPENDIX}

Matrix T. Matrix $\mathbf{T} \in \mathbb{R}^{6 n \times r}$ can be partitioned in $(6 \times 1)$-blocks $\mathbf{T}_{i, j}, i=1, \ldots, n, j=1, \ldots, r$, that can be evaluated by the following rule:

- if the tendon $j$ is not connected to the $i$-th link and does not pass through a sheath fixed to the $i$-th link, $\mathbf{T}_{i, j}=\mathbf{0}_{6 \times 1}$

- if the tendon $j$ is connected to the $i$-th link and successively passes through a sheath fixed to the $k$-th link,

$$
\mathbf{T}_{i, j}=\frac{1}{\left\|\mathbf{v}_{k, j}-\mathbf{v}_{i, j}\right\|}\left(\begin{array}{c}
\mathbf{v}_{k, j}-\mathbf{v}_{i, j} \\
\mathbf{v}_{i, j} \times \mathbf{v}_{k, j}
\end{array}\right)
$$

- if the tendon $j$ is connected to (or passes through) link $h$, passes through link $i$ and is connected to (or passes through) link $k$,

$$
\mathbf{T}_{i, j}=\frac{1}{\left\|\mathbf{v}_{h, j}+\mathbf{v}_{k, j}-2 \mathbf{v}_{i, j}\right\|}\left(\begin{array}{c}
\mathbf{v}_{h, j}+\mathbf{v}_{k, j}-2 \mathbf{v}_{i, j} \\
\mathbf{v}_{i, j} \times\left(\mathbf{v}_{h, j}+\mathbf{v}_{k, j}\right)
\end{array}\right) .
$$

Matrix H. Matrix $\mathbf{H} \in \mathbb{R}^{s \times 6 n^{2}}$ is nested block-diagonal, with diagonal blocks $\mathbf{H}_{i} \in \mathbb{R}^{s_{i} \times 6 n}$ defined as $\mathbf{H}_{i}=$ $\operatorname{diag}\left[\mathbf{H}_{i, j}\right]_{1, j \neq i}^{n}$. Blocks $\mathbf{H}_{i, j} \in \mathbb{R}^{t_{i, j} \times 6}$ embody the motion constraints imposed by the particular type of connection between bodies $i$ and $j$. Common contact types are

$$
\begin{array}{ll}
\mathbf{H}_{i, j}=\mathbf{I}_{3} & \text { for "hard finger"; } \\
\mathbf{H}_{i, j}=\mathbf{I}_{6} & \text { for "complete costraint"; } \\
\mathbf{H}_{i, j}=\left(\begin{array}{l|c}
\mathbf{I}_{3} & \mathbf{0} \\
\hline \mathbf{0} & \mathbf{n}_{i, j}
\end{array}\right) & \text { for "soft finger"; }
\end{array}
$$

where $\mathbf{n}_{i, j}$ is the direction of the normal between the objects $i$ and $j$. Another important type of connection is the "hinge" joint that only allows relative rotation around the axis $\mathbf{n}_{i, j}$ :

$$
\mathbf{H}_{i, j}=\left(\begin{array}{c|c}
\mathbf{I}_{3} & \mathbf{0} \\
\hline \mathbf{0} & \mathbf{a}_{i, j} \\
& \mathbf{b}_{i, j}
\end{array}\right)
$$

where $\mathbf{a}_{i, j}$ e $\mathbf{b}_{i, j}$ form a basis of the plane perpendicular to the direction $\mathbf{n}_{i, j}$.

Matrix K. The stiffness matrix $\mathbf{K} \in \mathbb{R}^{s \times s}$ has the same block structure as $\mathbf{H}$. Blocks $\mathbf{K}_{i, j} \in \mathbb{R}^{t_{i, j} \times t_{i, j}}$ are diagonal, and depend upon the type of contact and the stiffness of the virtual spring interposed between objects $i$ and $j$. In 
the easiest cases the elements of the matrix $\mathbf{K}_{i, j}$ are equal to $\mu_{t}$ for linear springs and to $\mu_{r}$ for rotoidal springs. Some examples follow:

$$
\begin{array}{ll}
\mathbf{K}_{i, j}=\mu_{t} \mathbf{I}_{3} & \text { for "hard finger"; } \\
\mathbf{K}_{i, j}=\left(\begin{array}{c|c}
\mu_{t} \mathbf{I}_{3} & \mathbf{0} \\
\hline \mathbf{0} & \mu_{r} \mathbf{I}_{3}
\end{array}\right) & \text { for "complete costraint"; } \\
\mathbf{K}_{i, j}=\left(\begin{array}{c|c}
\mu_{t} \mathbf{I}_{3} & \mathbf{0} \\
\hline \mathbf{0} & \mu_{r}
\end{array}\right) & \text { for "soft finger"; } \\
\mathbf{K}_{i, j}=\left(\begin{array}{c|c}
\mu_{t} \mathbf{I}_{3} & \mathbf{0} \\
\hline \mathbf{0} & \mu_{r} \mathbf{I}_{2}
\end{array}\right) & \text { for "hinge" joints; }
\end{array}
$$

$\mathbf{K}_{r} \in \mathbb{R}^{r \times r}$, the matrix of tendon's elasticity, is also diagonal.

Matrix X: The selection matrix $\mathbf{X} \in \mathbb{R}^{6 n^{2} \times 6 n^{2}}$ can be partitioned as follows:

$$
\mathbf{X}=\left(\begin{array}{ccc}
\mathbf{X}_{1,1} & \ldots & \mathbf{X}_{1, n} \\
\ldots & \ldots & \ldots \\
\mathbf{X}_{n, 1} & \ldots & \mathbf{X}_{n, n}
\end{array}\right)
$$

where the blocks $\mathbf{X}_{i, j} \in \mathbb{R}^{6 n \times 6 n}$ are defined as:

$$
\mathbf{X}_{i, j}=\left(\begin{array}{ccc}
\mathbf{X}_{i, j}^{1,1} & \ldots & \mathbf{X}_{i, j}^{1, n} \\
\ldots & \ldots & \cdots \\
\mathbf{X}_{i, j}^{n, 1} & \ldots & \mathbf{X}_{i, j}^{n, n}
\end{array}\right)
$$

- If $i=j$ (diagonal blocks), $\mathbf{X}_{i, i}$ is evaluated according to the following rules:

- blocks $\mathbf{X}_{i, i}^{h, k}$ with $h \neq k$ (non diagonal blocks) are null $6 \times 6$ matrices:

- blocks $\mathbf{X}_{i, i}^{h, h}$ (diagonal blocks):

$*$ if $i$-th object is in contact with the environment, $\mathbf{X}_{i, i}^{1,1}=$

$\mathbf{I}_{6} ;$

$*$ if $i$-th object is in contact with $j$-th object:

- if $i>j, \mathbf{X}_{i, i}^{j, j}=\mathbf{I}_{6}$;

- if $i<j, \mathbf{X}_{i, i}^{(j+1),(j+1)}=\mathbf{I}_{6}$;

- If $i \neq j$ (non diagonal block) matrix $\mathbf{X}_{i, j}$ :

- if $i$-th object is not in contact with $j$-th object, $\mathbf{X}_{i, j}=$ $\mathbf{0}_{6 n \times 6 n}$;

- if $i$-th object is in contact with $j$-th object then just one block of $\mathbf{X}_{i, j}$ is not void. In particular we have:

$*$ if $j>i \mathbf{X}_{i, j}^{j,(i+1)}=-\mathbf{I}_{6}$;
$*$ if $j<i \mathbf{X}_{i, j}^{(j+1), i}=-\mathbf{I}_{6}$.

- blocks which do not hold the above properties are null $6 \times 6$ matrices.

\section{REFERENCES}

[1] S. Jacobsen, H. Ko, E. Iversen, and C. Davis, "Antagonistic control of a tendon driven manipulator," in Proceedings IEEE International Conference on Robotics and Automation, 1989, pp. 1334-1339.

[2] A. Morecky, Z. Busko, H. Gastzold, and K. Javorek, "Synthesis and control of the anthropomorphic two-handed manipulator," in Proc. of the 10th Int. Symp. Industr. Robots, Milan, Italy, 1980, pp. $71-77$.

[3] J.K. Salisbury and B. Roth, "Kinematic and force analysis of articulated mechanical hands," J. Mech. Transm. Automat. in Des., vol. 105, pp. 35-41, 1983.

[4] R. Mukherjee, G. Song, and R. Satava, "An articulated instrument for enhanced dexterity in minimally invasive surgery," in Proc. 18th Annual International Conference of the IEEE Engineering in Medicine and Biology Society, 1997, vol. 1, pp. 220222.

[5] C. Melchiorri and G. Vassura, "A performance index for underactuated, multi-wire haptic interfaces," in Proceedings IEEE International Conference on Robotics and Automation, 1998, pp. 1026-1031.

[6] J. Lee and L. Tsai, "The structural synthesis of tendon-driven manipulators having a pseudotriangular structure matrix," International Journal of Robotics Research, vol. 10, no. 3, pp. 255-262, 1991.

[7] L. Barbieri and M. Bergamasco, "Nets of tendons and actuators: an anthropomorphic model for the actuation system of dextrous robot hands," in Proc. of 5th Int. Conf. Advanced Robotics, 1991, vol. 1, pp. 357-362.

[8] C.R. Johnstun and C.C. Smith, "Modelling and design of a mechanical tendon actuation system," ASME Transactions Journal on Dynamics, System, Measurements and Control, pp. 760-767, June 1992 .

[9] Y. Shen, H. Osumi, and T. Arai, "Set of manipulating forces in wire driven systems," in Proc. Int. Conf. on Int. Rob. Syst., Munich, Germany, 1994, vol. 3, pp. 1626-1631.

[10] R. Kurtz and V. Hayward, "Dexterity measures with unilateral actuation constraints: the $\mathrm{n}+1$ case," Journal of Advanced Robotics, vol. 9, no. 5, pp. 561-577, 1995.

[11] V. Hayward and J.M. Cruz-Hernandez, "Parameter sensitivity analysis for design and control of tendon transmission," in Experimental Robotics, Lecture Notes in Control and Information Sciences 223, pp. 83-90. Springer-Verlag London, 1995.

[12] K. Hyodo H. Kobayashi and D. Ogane, "On tendon-driven robotic mechanisms with redundant tendons," International Journal of Robotics Research, vol. 17, no. 5, pp. 561-571, 1996.

[13] H. Kobayashi, K. Hyodo, and D. Ogane, "On intelligent control of tendon-driven robotic mechanisms with redundant tendons," in Proc. 3rd MOVIC, Chiba: JSME, 1999, pp. 210-215.

[14] G.M. Prisco and F. Bergamasco, "Dynamic modeling of a class of tendon driven manipulators," in Proc. 8th Int. Conf. on Advanced Robotics, Monterey, CA, July 1997, pp. 893-899.

[15] F. Mussa Ivaldi, P. Morasso, and R. Zaccaria, "Kinematics network," B. Cybernetics, vol. 60, pp. 1-16, 1988.

[16] H. Buchner, M. Hines, and H. Hemani, "A dynamic model for finger interphalangeal coordination," Journal of Biomechanics, vol. 21 , pp. 459-468, 1988.

[17] D.C. Harding, K.D. Brandt, and B.M. Hillberry, "Finger joint force minimization in pianists using optimization techniques," Journal of Biomechanics, vol. 26, no. 12, pp. 1403-1412, 1993.

[18] J.N.A.L. Leijnse and J.J. Kalker, "A two-dimensional kinematic model of the lumbrical in the human finger," Journal of Biomechanics, vol. 28, no. 3, pp. 237-249, 1995.

[19] K. Hara, R. Yokogawa, and A. Yokogawa, "A graphical method for evaluating static characteristics of the human finger by force manipulability," in Proceedings IEEE International Conference on Robotics and Automation, 1998, pp. 1623-1628.

[20] J. Backera, N. Thakor, and K. Gruber, "A study of human hand tendon kinematics with applications to robot hand design," in Proceedings IEEE International Conference on Robotics and Automation, 1986, pp. 1540-1545.

[21] D. Deno, M. Murray, K. Pister, and S. Sastry, "Fingerlike biomedical robot," in Proceedings IEEE International Conference on Robotics and Automation, 1992, pp. 566-572.

[22] R.M. Kolacinski and R.D. Quinn, "Design and mechanics of an antagonistic biomimetic actuator system," in Proceedings IEEE International Conference on Robotics and Automation, 1998, pp. 1629-1634. 
[23] Y. K. Lee and I. Shimoyama, "A skeletal framework artificial hand actuated by pneumatic artificial muscles," in Proceedings IEEE International Conference on Robotics and Automation, 1999, pp. 926-935.

[24] J. Wittemburg, Dynamics of Systems of Rigid Bodies, B.G. Teubner, Stuttgart, 1977.

[25] A. Bicchi, "Force distribution in multiple whole-limb manipulation," in Proceedings IEEE International Conference on Robotics and Automation, 1993, pp. 196-201.

[26] A. Bicchi, J.K.Salisbury, and D.L. Brock, "Contact sensing from force and torque measurements," International Journal of Robotics Research, vol. 12, no. 3, pp. 249-262, 1993.

[27] A. Bicchi, "On the closure properties of robotic grasping," International Journal of Robotics Research, vol. 14, no. 4, pp. 319-334, 1995. 